INPLASY

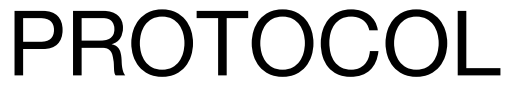

To cite: Ferreira et al.

Complications in the surgical treatment of Eagle syndrome:

A systematic review. Inplasy

protocol 202090072. doi:

10.37766/inplasy2020.9.0072

Received: 19 September 2020

Published: 19 September 2020

Corresponding author:

Mário Ferreira

mario.ferreira@docente.unieva

ngelica.edu.br

Author Affiliation:

UniEVANGÉLICA

Support: Personal support.

Review Stage at time of this submission: Piloting of the study selection process.

Conflicts of interest:

None.

\section{Complications in the surgical treatment of Eagle syndrome: A systematic review}

Ferreira, MS1; Miranda, G2; Almeida, FT3; Gasperini, G4; Silva, BSF5; Valladares-Neto, J6; Silva, MAG7; Santiago-Júnior, JF8.

Review question / Objective: What is the prevalence of complications from surgical approaches for the removal of the stylohyoid ligament in patients with Eagle syndrome?

Condition being studied: Eagle syndrome is a rare condition caused by an elongated or disfigured styloid process, which interferes with the functioning of neighboring structures and gives rise to orofacial and cervical pain often triggered by neck movements. Surgical shortening of the styloid process can be done through either an intraoral or external approach. There is no consensus in the literature which approach is better. This systematic review aims to demonstrate the prevalence of complications related to each approach, demonstrating the possible superiority of one in comparison to another.

INPLASY registration number: This protocol was registered with the International Platform of Registered Systematic Review and Meta-Analysis Protocols (INPLASY) on 19 September 2020 and was last updated on 19 September 2020 (registration number INPLASY202090072).

\section{INTRODUCTION}

Review question / Objective: What is the prevalence of complications from surgical approaches for the removal of the stylohyoid ligament in patients with Eagle syndrome?

Condition being studied: Eagle syndrome is a rare condition caused by an elongated or disfigured styloid process, which interferes 
with the functioning of neighboring structures and gives rise to orofacial and cervical pain often triggered by neck movements. Surgical shortening of the styloid process can be done through either an intraoral or external approach. There is no consensus in the literature which approach is better. This systematic review aims to demonstrate the prevalence of complications related to each approach, demonstrating the possible superiority of one in comparison to another.

\section{METHODS}

Search strategy: ( " Eagle syndrome"[Supplementary Concept] OR "Eagle syndrome" OR "Eagle's syndrome" OR "stylohyoid syndrome" OR "stylohyoid" OR "styloid syndrome" OR "styloid process syndrome" OR "elongated styloid" OR "disfigured styloid") ) AND ("surgery"[Subheading] OR "surgery"[TI/ AB] OR "surgeries"[TI/AB] OR "surgical" $[T I / A B]$ OR "Surgical Procedures, Operative"[Mesh] OR "operative"[TI/AB] OR "Intraoperative Complications"[Mesh] OR "Intraoperative" OR "removal" OR "removed").

Participant or population: Patients with eagle Syndrome.

Intervention: Stylohyoid ligament removal surgery.

Comparator: Any complications of surgical treatment.

Study designs to be included: Case series; Case report; observational studies.

Eligibility criteria: We will include studies that report the trans operative and postoperative complications arising from surgical approach. No restriction on publications period or language will be applied.

Information sources: Eletronic databases ( Pubmed/Medline; Embase; Lilacs; Web of Science; Scopus; Livivo; Cochrane); Grey Literature (Google Scholar; Proquest Dissertations; OpenGrey); Additional
Search ( Hand-searches of reference list from include studies; experts)

Main outcome(s): The response variable is the identification of any complications compared with external or intra-oral approach.

Additional outcome(s): Other variables of interest will be: Name, date of publication, field of the journal, country, number of patients, initial symptoms, type of access used, size of the procedure removed, complications, monitoring and status.

Data management: Two independent reviewers (1R and $2 R$ ) will select the included articles. First, in phase-one, both reviewers will read titles and abstracts independently while applying the eligibility criteria. Second, in phase-two, the same two reviewers (1R and $2 R$ ) will perform a full-text reading while applying the election criteria. In both phases, all the retrieved information will be crosschecked by the third review (3R). Final selection will be always based on the full-text of the publication. Data management with Endnote.

Quality assessment / Risk of bias analysis: Study methodological quality will be measured using Joanna Briggs Institute risk of bias using Checklist for case reports Checklist for case series; Checklist for prevalence studies. Two independent reviewers will assess the methodological quality for all included studies, respectively. A third expert investigator will help to solve all disagreements occur between two investigators.

Strategy of data synthesis: The synthesis will be carried out descriptively on the included studies with focuses: characteristics of the primary studies; characteristics of the sample studied; risk of bias; comparisons of the intra-oral and external approach and type of complications. The papers' findings will be described and figures or tables will be made to summarize and/or compare the information. Meta-analysis will be made 
with the information about complications and approach.

Subgroup analysis: None subgroup planned.

Sensibility analysis: If sufficient available data are extracted, we will plan to conduct sensitivity analysis to check the stability for the outcome results by excluding low methodological quality studies.

Language: Any article published in Latin Roman alphabet.

Country(ies) involved: Brazil.

Keywords: Eagle syndrome; surgical procedures; elongated styloid syndrome; intraoperative complications.

Dissemination plans: We will submit this study to a peer-reviewed journal for publication.

Contributions of each author:

Author 1 - Mário Ferreira - First Reviewer.

Author 2 - Geovane Miranda - Second Reviewer.

Author 3 - Fabiana Almeida - Third

Reviewer.

Author 4 - Giovanni Gasperini - Expert in field.

Author 5 - Brunno Silva - Expert in Systematic Review.

Author 6 - José Valladares-Neto - SubCoordinator.

Author 7 - Maria Silva - Coordinator.

Author 8 - Joel Santiago-Juníor - Statistic and analysis data. 